\title{
A CHARACTERIZATION OF CONFORMALLY FLAT SPACES
}

\section{A. H. TAUB ${ }^{1}$}

1. Introduction. The purpose of this paper is to show some analogies between the theory of groups of infinitesimal conformal transformations and that of groups of motions in Riemannian spaces. The main part of the paper will be devoted to Theorem I: The necessary and sufficient condition for an $n$-dimensional $(n \geqq 3)$ Riemannian space, $V_{n}$, to admit an $(n+1)(n+2) / 2$ parameter group of infinitesimal conformal transformations is that the $V_{n}$ be conformally flat.

This theorem is the analogue of the well known theorem concerning groups of motions of $n$-dimensional Riemannian spaces $V_{n}$, namely, ${ }^{2}$ $A$ group of motions of $a V_{n}$ has at most $n(n+1) / 2$ parameters and this number only in case $V_{n}$ has constant curvature. Sasaki ${ }^{3}$ has shown part of Theorem I by using the formalism of the conformal connection. He has shown that a space with a conformal connection will admit a maximal number of independent infinitesimal conformal transformations only if it is conformally flat but has not given this number. The proof given here of Theorem I will be carried out in a fashion similar to Eisenhart's proof of the latter one. We shall use the notation of R. G. throughout this paper.

In a $V_{n}(n>3)$ for which the Weyl conformal curvature tensor vanishes, there exists a coordinate system ${ }^{4}$ where

$$
g_{i j}=e^{2 \sigma(x)} e_{i} \delta_{i j}, \quad \dot{e}_{i}= \pm 1 .
$$

That is, the $V_{n}$ is conformal to a flat space $S_{n}$ with the metric

$$
a_{i j}=e_{i} \delta_{i j} \text {. }
$$

The $S_{n}$ admits $^{5}$ the conformal group of $(n+1)(n+2) / 2$ parameters generated by dilatation, inversions, translations and orthogonal transformations. The part of this group connected to the identity is

Received by the editors February 21, 1948.

${ }^{1}$ Guggenheim post-Service Fellow at the Institute for Advanced Study, on leave from the University of Washington.

${ }^{2}$ L. P. Eisenhart, Riemannian geometry, Princeton University Press, 1926, p. 239. This work will be referred to as R. G. hereafter.

${ }^{3}$ Shigeo Sasaki, Geometry of the conformal connexion, Science Reports of the Tôhoku Imperial University (1) vol. 29 (1940) pp. 219-267. I am indebted to J. L. Vanderslice for this reference and a valuable suggestion regarding the integrability condition of equation (3.5).

${ }^{4}$ R. G. p. 92.

'R. G. p. 94, problem 16. 
generated by an infinitesimal group of $(n+1)(n+2) / 2$ parameters. This infinitesimal group is simply isomorphic to a group in $V_{n}$ which is a group of infinitesimal conformal transformations of $V_{n}$ into itself. Hence if a $V_{n}(n \geqq 3)$ is conformally flat, it admits an $(n+1)(n+2) / 2$ parameter group of infinitesimal conformal transformations.

2. Infinitesimal conformal transformations. The proof of Theorem I will be completed by examining the differential equations satisfied by the vectors determining an infinitesimal conformal transformation of a $V_{n}$. These are ${ }^{6}$

$$
h_{i j}=\xi_{i, j}+\xi_{j, i}=\psi g_{i j}
$$

where the comma denotes covariant differentiation.

It is obvious from these equations that if $\xi_{\alpha \mid i}$ and $\xi_{\beta \mid i}$ are solutions with $\psi=\psi_{\alpha}$ and $\psi=\psi_{\beta}$ respectively then so is $\xi_{i}=a^{\alpha} \xi_{\alpha \mid i}+a^{\beta} \xi_{\beta \mid i}$ ( $\alpha$ and $\beta$ not summed) where $a^{\alpha}$ and $a^{\beta}$ are arbitrary constants and $\psi=a^{\alpha} \psi_{\alpha}+a^{\beta} \psi_{\beta}$. Hence we have ${ }^{7}$

If $\xi_{\sigma \mid i}$ for $\sigma=1, \cdots, r$ are components of infinitesimal conformal transformations of $a V_{n}$ so are $a^{\sigma} \xi_{\sigma \mid i}$, where the a's are arbitrary constants.

A straightforward calculation using (2.2) given below shows that under the same hypothesis as above

$$
\xi_{\beta \alpha \mid i}=\xi_{\beta \mid}^{j} \frac{\partial \xi_{\alpha \mid i}}{\partial x^{j}}-\xi_{\alpha \mid}^{j} \frac{\partial \xi_{\beta \mid i}}{\partial x^{j}}=\xi_{\beta \mid}^{j} \xi_{\alpha \mid i, j}-\xi_{\alpha \mid}^{j} \xi_{\beta \mid i, j}
$$

is also a solution of (2.1) with $\psi=\psi_{\beta \alpha}=\xi_{\beta \mid}^{k} \psi_{\alpha, k}-\xi_{\beta \mid}^{k} \psi_{\beta, k}$. Thus we have the result: ${ }^{7}$ If $X_{\sigma} f \equiv \xi_{\sigma}^{i} \partial f / \partial x^{i}$ for $\sigma=1,2, \cdots, r$ are the generators of infinitesimal conformal transformations of a $V_{n}$, so are $\left(X_{\sigma}, X_{\tau}\right)$ f for $\sigma, \tau=1, \cdots, r(\sigma \neq \tau)$.

These two results show that the set of all generators of infinitesimal conformal transformations of $V_{n}$ are closed under the operations of taking linear combinations and forming commutators, that is, these generators form a Lie algebra just as in the case of generators of motions of $V_{n}$.

From equation (2.1) it follows that

$$
h_{i j, k}+h_{i k, j}-h_{j k, i}=g_{i j} \psi_{, k}+g_{i k} \psi_{, j}-g_{j k} \psi_{, i} .
$$

By using the procedure given in R. G. it may be shown that

$$
\xi_{i, j k}=-\xi_{m} R_{k i j}^{m}+2^{-1}\left(\psi_{, k} g_{i j}+\psi_{, j} g_{i k}-\psi_{, i} g_{j k}\right)
$$

6 R. G. p. 231, equation (69.1).

7 Cf. R. G. $\$ 71$ p. 237. 
and the integrability conditions of these equations are

$$
\begin{aligned}
& \xi_{m}\left(R_{k i j, l}^{m}-R_{l i j, k}^{m}\right)+\xi_{m, l} R_{k i j}^{m}-\xi_{m, k} R_{l i j}^{m}+\xi_{i, m} R_{j k l}^{m}+\xi_{m, j} R_{i k l}^{m} \\
& +2^{-1}\left(g_{i l} \psi_{, j k}-g_{i k} \psi_{, j l}+g_{j k} \psi_{, i l}-g_{j l} \psi, i k\right)=0 \text {. }
\end{aligned}
$$

3. Transformation of the integrability conditions. We now transform equations (2.3) into another form. From the Bianchi identity

$$
R_{h i j k, l}+R_{h i k l, j}+R_{h i l j, k}=0
$$

it follows that equation (2.3) may be written as

$$
\begin{aligned}
-\xi^{m} R_{i j k l, m}+\xi_{m, l} R_{k i j}^{m}-\xi_{m, k} R_{l i j}^{m}+\xi_{i, m} R_{j k l}^{m}+\xi_{m, j} R_{i k l}^{m} \\
+2^{-1}\left(g_{i l} \psi_{, j k}-g_{i k} \psi_{, j l}+g_{j k} \psi_{, i l}-g_{j l} \psi, i k\right)=0 .
\end{aligned}
$$

To eliminate $\psi$ from equation (3.2) we multiply by $g^{i l}$ and sum. The resulting expression is multiplied by $g^{j k}$ and summed. We thus obtain

(3.3) $-\xi^{m} R_{j k, m}-\xi_{m, k} R_{j}^{m}-\xi_{m, j} R_{k}^{m}+2^{-1}(n-2) \psi_{, j k}+2^{-1} g_{j k} \Delta_{2} \psi=0$,

$$
\Delta_{2} \psi=\frac{1}{n-1}\left(\xi^{m} R_{, m}+2 \xi_{m, k} R^{m k}\right)
$$

where $\Delta_{2} \psi=g^{i j} \psi_{, i j}$. Substituting for $\Delta_{2} \psi$ from (3.4) into (3.3) we obtain

$$
\begin{aligned}
\frac{1}{2}(n-2) \psi_{, j k}= & \xi^{m}\left(R_{j k, m}-\frac{1}{2(n-1)} g_{j k} R_{, m}\right) \\
& +\xi_{m, k} R_{j}^{m}+\xi_{m, j} R_{k}^{m}-\frac{1}{(n-1)} g_{i k} \xi_{m, l} R^{m l}
\end{aligned}
$$

When this value of $\psi_{, j k}$ is substituted into (3.2) we obtain

$$
-\xi^{m} C_{i j k l, m}+\xi_{m, p}\left(\delta_{l}^{p} C_{k i j}^{m}-\delta_{k}^{p} C_{l i j}^{m}+\delta_{i}^{m} C_{j k l}^{p}+\delta_{j}^{p} C_{i k l}^{m}\right)=0
$$

as the integrability conditions of equations (2.2) where $C_{i j k}^{h}$ is the Weyl conformal curvature tensor and is defined in terms of the Riemann tensor, Ricci tensor and the scalar curvature by

$$
\begin{gathered}
C_{i j k}^{h}=R_{i j k}^{h}+\frac{1}{n-2}\left(\delta_{j}^{h} R_{i k}-\delta_{k}^{h} R_{i j}+g_{i k} R_{j}^{h}-g_{i j} R_{k}^{h}\right) \\
+\frac{R}{(n-1)(n-2)}\left(\delta_{k}^{h} g_{i j}-\delta_{j}^{h} g_{i k}\right) .
\end{gathered}
$$


The integrability conditions of equations (3.5) are

$$
\xi^{m} R_{j k l, m}+\xi_{, l}^{m} R_{j k m}-\xi_{, k}^{m} R_{j l m}-2^{-1}(n-2) \psi_{, p} C_{j k l}^{p}=0
$$

where

$$
R_{j k l}=\left(R_{j k}-\frac{R}{2(n-1)} g_{j k}\right)_{, l}-\left(R_{j l}-\frac{R}{2(n-1)} g_{j l}\right)_{, k} .
$$

Equations (2.2), (3.5) and the equations

$$
\frac{\partial \xi_{i}}{\partial x^{j}}=\xi_{i, j}+\xi_{m}\left\{\begin{array}{l}
m \\
i j
\end{array}\right\}, \quad \frac{\partial \psi}{\partial x^{i}}=\psi_{, i}
$$

constitute a system of equations of the form

$$
\frac{\partial \theta^{\alpha}}{\partial x^{i}}=\psi_{i}^{\alpha}(x, \theta), \quad \alpha=1,2, \cdots,(n+1)^{2} ; i=1,2, \cdots, n,
$$

where $\theta^{\alpha}$ represents the set of $(n+1)^{2}$ functions $\psi ; \xi_{i} ; \psi_{, i}$; and $\xi_{i, j}$. The functions $\theta^{\alpha}$ are subject to the $n(n+1) / 2$ algebraic relations $(2.1)$. The system (3.10) is of a standard form ${ }^{8}$ and it is well known that the maximum number of linearly independent $\xi_{i}$ is $(n+1)(n+2) / 2$ and that this number obtains when the integrability conditions of (3.10) are identically satisfied in virtue of (2.1). That is, if a space admits this maximum number of linearly independent infinitesimal conformal transformations equations (3.6) and (3.8) must be satisfied for arbitrary choices of the $\xi_{i}$ and $\xi_{i, j}$ satisfying (2.1). Thus

$$
C_{i j k l, m}=0
$$

and at least the antisymmetric part with respect to $p$ and $m$ of the coefficient of $\xi_{m, p}$ in equation (3.6) must vanish. That is, we must have

$$
\delta_{l}^{p} C_{k i j}^{m}-\delta_{k}^{p} C_{l i j}^{m}+\delta_{i}^{m} C_{j k l}^{p}+\delta_{j}^{p} C_{i k l}^{m}=\delta_{l}^{m} C_{k i j}^{p}-\delta_{k}^{m} C_{l i j}^{p}+\delta_{i}^{p} C_{j k l}^{m}+\delta_{j}^{m} C_{i k l}^{p} .
$$

Setting $p=l$, summing, and using the fact that $C_{i j m}^{m}=0$ we obtain

$$
(n-1) C_{k i j}^{m}=C_{i j k}^{m}+C_{j k i}^{m}+C_{k i j}^{m}=0
$$

the last equality being a consequence of the symmetry properties of $C_{i j k}^{m}$. Hence if a $(n+1)(n+2) / 2$ parameter group of infinitesimal conformal transformations is to exist we must have

${ }^{8}$ Cf. L. P. Eisenhart, Non-Riemannian geometry, Amer. Math. Soc. Colloquium Publications, vol. 8, New York, 1927, p. 14. 


$$
C_{k i j}^{m}=0 .
$$

This is the necessary and sufficient condition for a $V_{n}(n>3)$ to be conformally flat.

If $n>3$ then it follows from

$$
C_{i j k, h}^{h}=\frac{n-3}{n-2} R_{i j k}
$$

that equations (3.8) are satisfied when (3.11) holds. If $n=3$, equations (3.6) are automatically satisfied since the Weyl conformal curvature tensor vanishes in this case. If a $V_{3}$ admits a 10 parameter group of infinitesimal conformal transformations then the argument used above applied to (3.8) leads to

$$
R_{j k l, m}=0
$$

and

$$
g_{p l} R_{j k m}-g_{m l} R_{j k p}-g_{p k} R_{j l m}+g_{m k} R_{j l p}=0 .
$$

Multiplying the last equation by $g^{p l}$ and summing gives

$$
R_{j k m}=0 \text {. }
$$

This is the necessary and sufficient condition for a $V_{3}$ to be conformally flat.

INSTITUTE FOR ADVANCED STUdY 